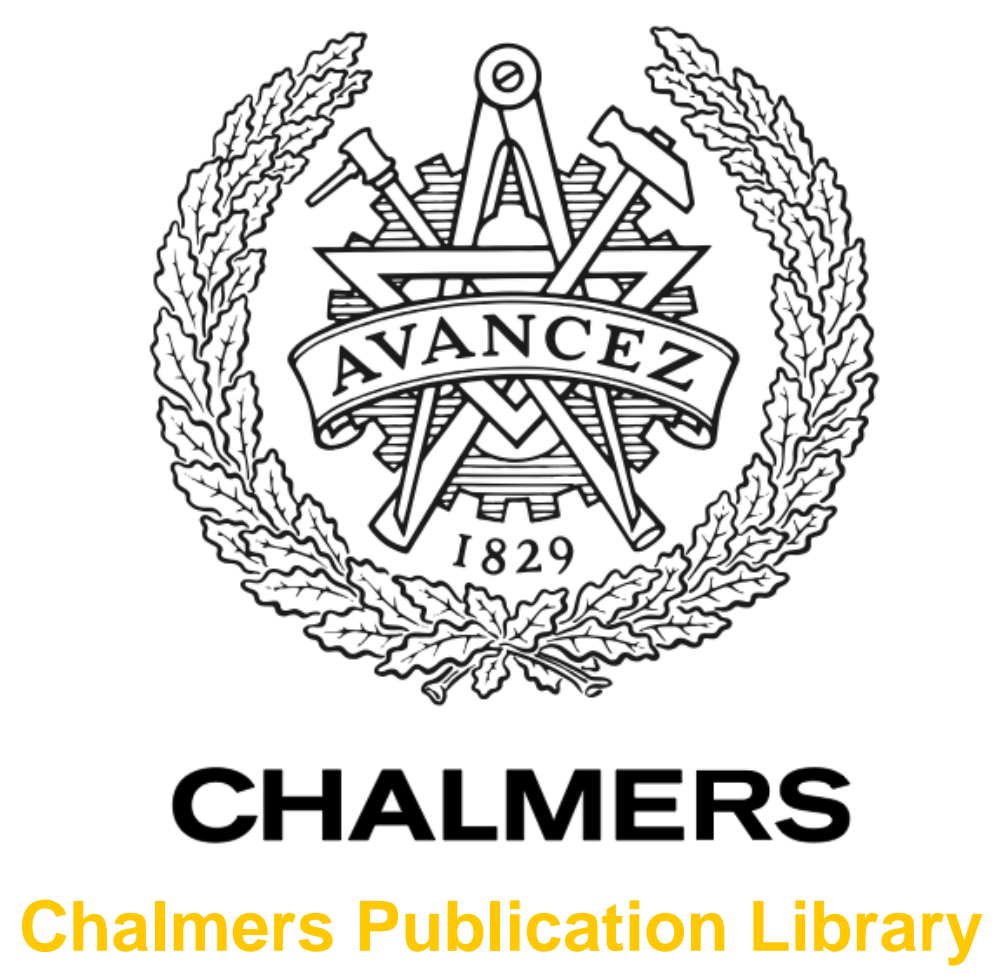

Location-Aided Pilot Contamination Elimination for Massive MIMO Systems

This document has been downloaded from Chalmers Publication Library (CPL). It is the author's version of a work that was accepted for publication in:

IEEE Global Communications Conference, Dec. 2015

Citation for the published paper:

Muppirisetty, L. ; Wymeersch, H. ; Karout, J. et al. (2015) "Location-Aided Pilot

Contamination Elimination for Massive MIMO Systems". IEEE Global Communications

Conference, Dec. 2015

Downloaded from: http://publications.lib.chalmers.se/publication/228139

Notice: Changes introduced as a result of publishing processes such as copy-editing and formatting may not be reflected in this document. For a definitive version of this work, please refer to the published source. Please note that access to the published version might require a subscription.

Chalmers Publication Library (CPL) offers the possibility of retrieving research publications produced at Chalmers University of Technology. It covers all types of publications: articles, dissertations, licentiate theses, masters theses, conference papers, reports etc. Since 2006 it is the official tool for Chalmers official publication statistics. To ensure that Chalmers research results are disseminated as widely as possible, an Open Access Policy has been adopted.

The CPL service is administrated and maintained by Chalmers Library. 


\title{
Location-Aided Pilot Contamination Elimination for Massive MIMO Systems
}

\author{
L. Srikar Muppirisetty*, Henk Wymeersch*, Johnny Karout ${ }^{\dagger}$, and Gabor Fodor ${ }^{\dagger}$ \\ *Department of Signals and Systems, Chalmers University of Technology, Gothenburg, Sweden \\ ${ }^{\dagger}$ Ericsson Research, Stockholm, Sweden \\ E-mail: \{ srikar.muppirisetty, henkw\}@ chalmers.se, \{johnny.karout, gabor.fodor\}@ ericsson.com
}

\begin{abstract}
Massive MIMO systems, while being a promising technology for $5 \mathrm{G}$ systems, face a number of practical challenges. Among those, pilot contamination stands out as a key bottleneck to design high-capacity beamforming methods. We propose and analyze a location-aided approach to reduce the pilot contamination effect in uplink channel estimation for massive MIMO systems. The proposed method exploits the location of user terminals, scatterers, and base stations. The approach removes the need for direct estimation of large covariance matrices and provides good channel estimation performance in the large antenna regime.
\end{abstract}

\section{INTRODUCTION}

The use of very large antenna arrays at the base station (BS) is considered as a promising technology for $5 \mathrm{G}$ communications [1]. Such massive MIMO systems boost the spectral efficiency and lead to a uniform user experience, as opposed to large variations in the received user bit rate or quality of service between the cell center and cell edge [2]-[5]. The underlying theory of massive MIMO systems is that under the assumption of perfect channel estimation, the channel of a served user grows orthogonal to other users and thereby interference can be virtually eliminated [6].

Pilot sequences, which are necessary for channel estimation, are a scarce resource. This is due to the fact that the length, i.e., number of symbols, of pilot sequences is limited by the coherence time and bandwidth of the wireless channel. In turn, the number of separable users is limited by the number of the available orthogonal pilot sequences. Consequently, when the number of antennas grows large, the number of spatially separable users is not limited by the number of antennas but by the number of available orthogonal pilot sequences [4], [5]. Therefore, in multicell systems, the pilot sequences must be reused, which leads to interference between identical pilot sequences from users in neighboring cells. This interference in multicell massive MIMO systems is known as the pilot contamination problem [7]. Pilot contamination is known to degrade the quality of channel state information at the BS, which in turn degrades the performance in terms of achieved spectral efficiency, beamforming gains, and cell edge user throughput.

Existing approaches to mitigate pilot contamination include blind channel estimation using eigenvalue-decomposition [8], greater-than-one pilot reuse schemes [9], modifying the frame structure such that the pilots are transmitted in each cell in non-overlapping time slots [10], [11], transmitting pilots in consecutive phases in which each BS keeps silent in one phase and repeatedly transmits in other phases [12], mixture of downlink and uplink training [13], and exploiting secondorder statistics of desired and interfering user channels [14]. In particular, [14] exploited the covariance matrices of the desired and interfering channels and showed that by carefully allocating the pilots to the users, the pilot contamination effect is eliminated in the large antenna array regime. The design is based on the knowledge of the covariance matrices of the desired channel, as well as the interfering channels. These covariance matrices scale roughly quadratically with the number of antennas per user and thus may be impractical to estimate. Also, the covariance matrices of the users change with their mobility and with the environmental changes and the corresponding propagation conditions.

In this paper, we propose to use location information inspired from the works of [15]-[17], for determining the appropriate pilot sequence assignments to users. Based on the observation that long-term channel characteristics and thereby user separation (i.e., the establishment of a set of spatially separable users) can be related to user location, pilot sequence assignment can be arranged such that users that are spatially separated reuse identical pilot sequences. The proposed approach essentially eliminates the scalability problem in estimating covariance matrices, both in terms of number of antennas and number of users and network nodes. In particular, we relate the mean and the standard deviation of the angle-of-arrival (AoA) to a user location, rather than to a user's channel. Through numerical evaluations, we demonstrate the performance of the proposed method for different user selection methods. Our results indicate that the proposed method approaches interference-free channel estimation for the large antenna regime.

Notation: We denote vectors in bold $\mathbf{x}$, matrices in bold capitals $\mathbf{X}, \mathbf{X}^{\mathrm{T}}$ and $\mathbf{X}^{\mathrm{H}}$ denote transpose and hermitian, respectively. The $i$-th entry of $\mathbf{x}$ is denoted by $[\mathbf{x}]_{i} \cdot \operatorname{vec}[\mathbf{X}]$ denotes stacking row-wise all the elements of $\mathbf{X}$ in a vector. $U[a, b]$ denotes a uniform distribution over the interval $a<b$. The Kronecker product of two matrices $\mathbf{X}_{1}$ and $\mathbf{X}_{2}$ is denoted as $\mathbf{X}_{1} \otimes \mathbf{X}_{2} \cdot \mathbf{I}_{M}$ denotes the identity matrix of size $M \times M$ and $\|\cdot\|_{F}$ denotes the Frobenius norm. 


\section{System Model}

We consider a two-dimensional scenario with $L$ hexagonal cells, and each cell is served by one BS, which is equipped with $M$ antennas. Users are located uniformly within the cells. The location of the $i$-th user in the $j$-th cell is denoted by $\mathbf{x}_{i j}^{\mathrm{u}} \in \mathbb{R}^{2}$, while the location of the BS in $k$-th cell is written as $\mathbf{x}_{k}^{\mathrm{b}} \in \mathbb{R}^{2}$. The objective of the BS is to estimate the uplink channel from a desired user, in the presence of $L-1$ interfering users.

\section{A. Channel Model}

The uplink channel of user $i$ from cell $j$ to BS $k$ is denoted by $\mathbf{h}_{i j k} \in \mathbb{C}^{M}$. We note that the channel only depends on the user $i$ and the base station $k$, but the use of the additional index $j$ will allow us to distinguish users from their own base station $(j=k)$ with users from another base station $(j \neq k)$. The channel is modeled as the superposition of $P$ arriving paths

$$
\mathbf{h}_{i j k}=\frac{1}{\sqrt{P}} \sum_{p=1}^{P} \mathbf{a}\left(\theta_{i j k}^{(p)}\right) \alpha_{i j k}^{(p)},
$$

where $\mathbf{a}\left(\theta_{i j k}\right) \in \mathbb{C}^{M}$ is the antenna steering vector corresponding to AoA $\theta_{i j k} \in[0,2 \pi), p$ is the path index, and $\alpha_{i j k}^{(p)}$ is the channel coefficient of the $p$-th path. The AoA's are assumed to be i.i.d., with probability density $p\left(\theta_{i j k}\right)$. We model $\alpha_{i j k}^{(p)} \stackrel{\text { i.i.d. }}{\sim} \mathcal{C N}\left(0, \beta_{i j k}\right)$, in which $\beta_{i j k}$ depends on pathloss and shadowing between user $i$ in cell $j$ and BS $k$. In particular, let $\alpha$ be a constant that depends on cell edge signalto-noise ratio (SNR) and cell radius $R$. It is defined as

$$
\alpha[\mathrm{dB}]=\gamma_{\mathrm{SNR}}+10 \eta \log _{10}(R)+10 \log _{10}\left(\sigma_{n}^{2}\right),
$$

where $\gamma_{\mathrm{SNR}}$ is the cell edge SNR in $\mathrm{dB}, \eta$ is the path-loss exponent, and $\sigma_{n}^{2}$ is the receiver noise power in $\mathrm{dBm}$. Then, in linear scale $\beta_{i j k}=\alpha\left\|\mathbf{x}_{i j}^{\mathrm{u}}-\mathbf{x}_{k}^{\mathrm{b}}\right\|_{2}^{-\eta}$. Restricting ourselves to uniform linear arrays, the $m$-th entry in the steering vector is given by [14]

$$
\left[\mathbf{a}\left(\theta_{i j k}\right)\right]_{m}=\exp \left(-j 2 \pi m D \cos \left(\theta_{i j k}\right) / \lambda\right),
$$

in which $D$ is the antenna spacing at the BS and $\lambda$ is the signal wavelength. It should be noted that we can limit $\theta_{i j k} \in[0, \pi]$ because any angle $\theta_{i j k} \in[-\pi, 0]$ can be replaced by $-\theta_{i j k}$, thus giving the same steering vector.

\section{B. Received Pilot Signal}

Assume $L$ users, one at each BS, have been assigned the same pilot sequence s of length $\tau$. For notational convenience, we will further assume that all the users indexed with $i$ are assigned the same pilot sequence s. Later, in Section IV, we will present various ways to assign users across cells to a given pilot sequence. The received $M \times \tau$ pilot signal observed at BS $k$ is written as

$$
\mathbf{Y}_{k}=\mathbf{h}_{i k k} \mathbf{s}^{\mathrm{T}}+\sum_{j=1, j \neq k}^{L} \mathbf{h}_{i j k} \mathbf{s}^{\mathrm{T}}+\mathbf{N}
$$

where $\mathbf{N} \in \mathbb{C}^{M \times \tau}$ is spatially and temporally additive white Gaussian noise (AWGN) with element-wise variance $\sigma_{n}^{2}$. In (4), $\mathbf{h}_{i k k}$ is the desired signal channel in the cell $k$ and $\mathbf{h}_{i j k}$ $(j \neq k)$ are the channels of interfering signals from other cells.

\section{UPLink ChANNEl Estimation}

\section{A. MMSE Channel Estimator}

The minimum mean square error (MMSE) estimate of the desired channel $\mathbf{h}_{i k k}$ by BS $k$ is then given by [14, Eq. (18)]

$$
\hat{\mathbf{h}}_{i k k}=\mathbf{R}_{i k k}\left(\sigma^{2} \mathbf{I}_{M}+\tau \sum_{j=1}^{L} \mathbf{R}_{i j k}\right)^{-1} \overline{\mathbf{S}}^{\mathrm{H}} \operatorname{vec}\left[\mathbf{Y}_{k}\right],
$$

where $\overline{\mathbf{S}}=\mathbf{s} \otimes \mathbf{I}_{M}$ and $\mathbf{R}_{i j k} \in \mathbb{C}^{M \times M}$ is the covariance matrix of $\mathbf{h}_{i j k}$, given by

$$
\mathbf{R}_{i j k}=\beta_{i j k} \int p\left(\theta_{i j k}\right) \mathbf{a}\left(\theta_{i j k}\right) \mathbf{a}^{\mathrm{H}}\left(\theta_{i j k}\right) \mathrm{d} \theta_{i j k},
$$

due to (1) and the assumptions from Section II-A. The estimator (5) critically relies on the knowledge of the covariance matrices. Due to the high-dimensional nature of $\mathbf{R}_{i j k}$, this knowledge is difficult to obtain in practice. When $\mathbf{R}_{i j k}$ is perfectly known to $\mathrm{BS} k$, intelligent pilot assignment can be performed to minimize the effect of pilot contamination [14].

The channel estimate of the desired channel in the presence of no interfering signals from other cells can be obtained by setting the interference terms to zero in (5), leading to the estimate

$$
\hat{\mathbf{h}}_{i k k}^{\text {no-int }}=\mathbf{R}_{i k k}\left(\sigma^{2} \mathbf{I}_{M}+\tau \mathbf{R}_{i k k}\right)^{-1} \overline{\mathbf{S}}^{\mathrm{H}} \mathbf{y}_{k},
$$

where $\mathbf{y}_{k}=\overline{\mathbf{S}} \mathbf{h}_{i k k}+\operatorname{vec}[\mathbf{N}]$ is the received $\tau \times 1$ signal vector at the BS $k$ under no interference from the other cell users.

\section{B. Location-Aided Covariance Estimation}

The distribution $p\left(\theta_{i j k}\right)$ is governed by the physical propagation environment. In some scenarios, as in [18, Fig. 1], propagation can be dominated by scatterers in the vicinity of the users. As an approximation, we consider a ring of radius $r_{\mathrm{s}}$ comprising many scatterers around the user. In that case, $p\left(\theta_{i j k}\right)$ corresponds to a uniform distribution:

$$
\theta_{i j k} \sim U\left[\theta_{i j k}^{\min }, \theta_{i j k}^{\max }\right],
$$

for some fixed $\theta_{i j k}^{\min }<\theta_{i j k}^{\max } \in[0, \pi]$. In particular,

$$
\begin{aligned}
\theta_{i j k}^{\min } & =\arctan \left(\frac{\left[\mathbf{x}_{i j}^{\mathrm{u}}\right]_{2}-\left[\mathbf{x}_{k}^{\mathrm{b}}\right]_{2}}{\left[\mathbf{x}_{i j}^{\mathrm{u}}\right]_{1}-\left[\mathbf{x}_{k}^{\mathrm{b}}\right]_{1}}\right)-\arcsin \left(\frac{r_{\mathrm{s}}}{\left\|\mathbf{x}_{i j}^{\mathrm{u}}-\mathbf{x}_{k}^{\mathrm{b}}\right\|_{2}}\right), \\
\theta_{i j k}^{\max } & =\arctan \left(\frac{\left[\mathbf{x}_{i j}^{\mathrm{u}}\right]_{2}-\left[\mathbf{x}_{k}^{\mathrm{b}}\right]_{2}}{\left[\mathbf{x}_{i j}^{\mathrm{u}}\right]_{1}-\left[\mathbf{x}_{k}^{\mathrm{b}}\right]_{1}}\right)+\arcsin \left(\frac{r_{\mathrm{s}}}{\left\|\mathbf{x}_{i j}^{\mathrm{u}}-\mathbf{x}_{k}^{\mathrm{b}}\right\|_{2}}\right) .
\end{aligned}
$$

Combined with the knowledge of $\beta_{i j k}$, which can also be related to the user's position since $\beta_{i j k}=\alpha\left\|\mathbf{x}_{i j}^{\mathrm{u}}-\mathbf{x}_{k}^{\mathrm{b}}\right\|_{2}^{-\eta}$, it is then possible to numerically compute $\mathbf{R}_{i j k}$. Hence, given the location of the $L$ users, BS $k$ can infer the covariance matrices and utilize those to compute $\hat{\mathbf{h}}_{i k k}$ in (5). 


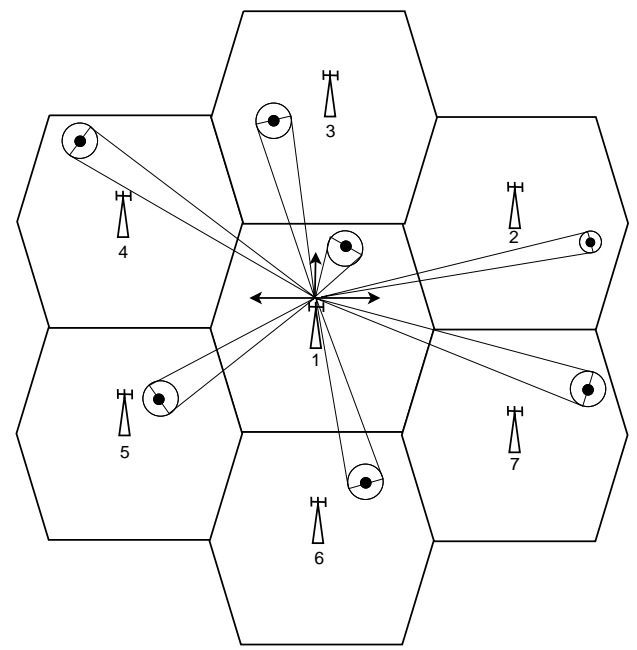

(a)

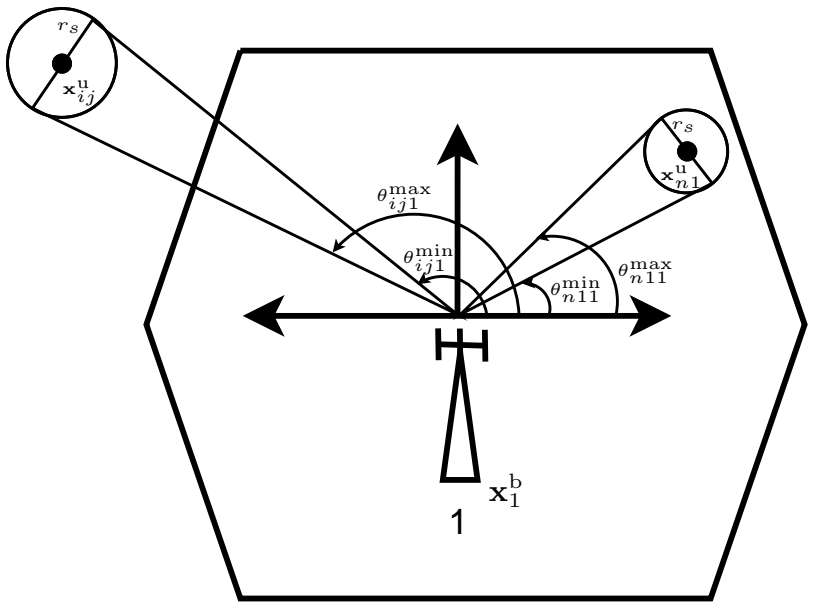

(b)

Fig. 1. (a) A two-dimensional 7-cell hexagonal layout with a BS placed at the center of each cell. The users in each cell are marked with a black dot and the scatterers lie within a circle around the user. The center cell (cell 1) is the target cell with the desired user and users from other cells (cells indexed 2 to 7) form the set of interfering users; (b) The support of AoA of desired and interfering users at the target BS and shows a specific example with desired user location $\mathbf{x}_{n 1}^{\mathrm{u}}$, target BS location $\mathbf{x}_{1}^{\mathrm{b}}$, interfering user location $\mathbf{x}_{i j}^{\mathrm{u}}$ and the radius of the ring of scatterers $r_{\mathrm{s}}$. The supports of the desired and interfering user's AoA at the target $\mathrm{BS}$ are $\left[\theta_{n 11}^{\min }, \theta_{n 11}^{\max }\right]$ and $\left[\theta_{i j 1}^{\min }, \theta_{i j 1}^{\max }\right]$, respectively.

\section{Location-Aided User SElection for Pilot CONTAMINATION REDUCTION}

In Section II-B, we considered $L$ arbitrary users that were assigned the same pilot sequence. However, using location information, a more informed assignment is possible. We consider the scenario of a given target user in cell 1, say user $n$, with channel $\mathbf{h}_{n 11}$ determined by AoA distribution $\left[\theta_{n 11}^{\min }, \theta_{n 11}^{\max }\right]$. Our objective is to find $L-1$ users in the surrounding cells and assign them the same pilot sequence as user $n$. These users have AoAs in the ranges $\left\{\left[\theta_{i j 1}^{\min }, \theta_{i j 1}^{\max }\right]\right\}_{j \neq 1}$ for the corresponding channels $\left\{\mathbf{h}_{i j 1}\right\}_{j \neq 1}$. It has been shown in $\left[14\right.$, Theorem 1] that when the intervals $\left\{\left[\theta_{i j 1}^{\min }, \theta_{i j 1}^{\max }\right]\right\}_{j \neq 1}$ are strictly non-overlapping with $\left[\theta_{n 11}^{\min }, \theta_{n 11}^{\max }\right]$, then

$$
\lim _{M \rightarrow \infty} \hat{\mathbf{h}}_{n 11}=\hat{\mathbf{h}}_{n 11}^{\text {no-int }} \text {. }
$$

The setup is visualized in Fig. 1, depicting the location of users, BSs, radius of scatterers, and the description of the user's AoA support.

We now consider two approaches for user assignment: random and location-based, both of which are detailed below.

\section{A. Random User Assignment}

In the random assignment, a single user is chosen randomly in each cell and assigned the same pilot sequence. A random assignment cannot always guarantee that $\left\{\left[\theta_{i j 1}^{\min }, \theta_{i j 1}^{\max }\right]\right\}_{j \neq 1}$ does not overlap with $\left[\theta_{n 11}^{\min }, \theta_{n 11}^{\max }\right]$, so that $\lim _{M \rightarrow \infty} \hat{\mathbf{h}}_{n 11} \neq$ $\hat{\mathbf{h}}_{n 11}^{\text {no-int }}$, which in turn implies that in the large-antenna regime, the estimate of the channel will be limited by interference.

\section{B. Location-Based User Assignment}

Under the assumption that we can estimate the covariance matrices $\mathbf{R}_{i j k}$ from user locations, the user assignment can be performed based on the location, accounting for the following conditions:

- Condition C1: The support of interfering signals AoA $\left\{\left[\theta_{i j 1}^{\min }, \theta_{i j 1}^{\max }\right]\right\}_{j \neq 1}$ should be strictly non-overlapping with the support of the desired signal AoA $\left[\theta_{n 11}^{\min }, \theta_{n 11}^{\max }\right]$;

- Condition C2: The support of interfering signal AoA $\left\{\left[\theta_{i j 1}^{\min }, \theta_{i j 1}^{\max }\right]\right\}_{j \neq 1}$ is maximally separated from the support of the desired signal AoA $\left[\theta_{n 11}^{\min }, \theta_{n 11}^{\max }\right]$; and

- Condition C3: The support of interfering signal AoA $\left\{\left[\theta_{i j 1}^{\min }, \theta_{i j 1}^{\max }\right]\right\}_{j \neq 1}$ is minimally overlapping with the support of the desired signal AoA $\left[\theta_{n 11}^{\min }, \theta_{n 11}^{\max }\right]$.

Condition $\mathrm{C} 1$ is based directly on the results from [14, Theorem 1] and ensures good channel estimation for $M \rightarrow \infty$. There may be multiple sets of users to satisfy $\mathrm{C} 1$. For that reason, we can optimize within all these sets, and impose Condition $\mathrm{C} 2$, which is stronger than $\mathrm{C} 1$, to ensure that the interference is as limited as possible. Finally, since in general $\mathrm{C} 1$ and thus C2 may not always be possible to satisfy, we also consider a weaker condition $\mathrm{C} 3$, which aims to minimize the interference, but cannot guarantee that (11) holds. The algorithm to select users based on conditions $\mathrm{C} 1, \mathrm{C} 2$, and $\mathrm{C} 3$ is described now.

Let $\mathbf{x}_{n 1}^{\mathrm{u}}$ be the location of the desired user in the center cell with support $\left[\theta_{n 11}^{\min }, \theta_{n 11}^{\max }\right]$. Let us assume each cell comprises $K$ users. Let us introduce the variables $y_{i j}^{(1)} \in\{0,1\}, y_{i j}^{(2)} \in$ $\{0,1\}$, where one of which takes on the value 1 if $i$-th user in $j$-th cell has been selected and 0 otherwise. The variables $y_{i j}^{(1)}$ and $y_{i j}^{(2)}$ distinguish whether the support of the interfering user's AoA is either to the left or right of the support of the desired user's AoA respectively. The user assignment can be written as an integer linear program: 
TABLE I

$$
\begin{aligned}
\underset{\left\{y_{i j}^{(1)}, y_{i j}^{(2)}\right\}_{i=1, \ldots K}^{j=2, \ldots L}}{\operatorname{maximize}} & \sum_{j=2}^{L} \sum_{i=1}^{K}\left(\left(\theta_{i j 1}^{\min }-\theta_{n 11}^{\max }\right) y_{i j}^{(1)}\right. \\
& \left.+\left(\theta_{n 11}^{\min }-\theta_{i j 1}^{\max }\right) y_{i j}^{(2)}\right) \\
\text { subject to } & \sum_{i=1}^{K} y_{i j}^{(1)}+y_{i j}^{(2)}=1, \forall j \\
& y_{i j}^{(1)} \in\{0,1\}, y_{i j}^{(2)} \in\{0,1\} .
\end{aligned}
$$

We note the following: (12a) maximizes the distance between the AoA supports of the desired and the interfering users which is based on condition C2; (12b) guarantees that only one user is selected from each cell; and (12c) imposes the binary integer requirements on the optimization variables.

The above optimization problem is always feasible. The problem (12) gives preference to users that satisfy the condition $\mathrm{C} 2$, in which case the objective is maximized. It might be possible that the user locations are such that neither C1 nor C2 can be satisfied. This is tackled in (12), as it implicitly considers the condition $\mathrm{C} 3$ in the formulation. For example, when the support of the interfering signal AoA $\left\{\left[\theta_{i j 1}^{\min }, \theta_{i j 1}^{\max }\right]\right\}_{j \neq 1}$ is overlapping with the support of the desired signal AoA $\left[\theta_{n 11}^{\min }, \theta_{n 11}^{\max }\right]$, the terms $\left(\theta_{i j 1}^{\min }-\theta_{n 11}^{\max }\right)$ and $\left(\theta_{n 11}^{\min }-\theta_{i j 1}^{\max }\right)$ in the objective function become negative. Therefore, to maximize the objective, the interfering users are selected in such way that provides minimal overlap with the support of the desired signal AoA.

\section{Remarks on Joint Assignment and Mutual Interference}

We note that both the random and location-based assignments consider one user in cell 1 . Hence, after a set of users has been assigned a certain pilot, the process is repeated for a second user in the center cell, and so forth. This is a greedy approach, which provides the most benefit for the first user, and less benefit for later users, as there will be fewer users from the other cells to choose from. Hence, a one-shot joint assignment of all users in the center cell could lead to better performance, but that is not the focus of this study.

We also note that the assignment aims to reduce the interference seen by the center cell users, with no regard to the interference that non-center cell users experience with respect to each other. Hence, a joint design across multiple cells is needed to benefit from the proposed scheme for all users in the system. This is also left as a topic for future work.

\section{Numerical Results}

We consider a 7-cell network with the center cell being the target cell, i.e., cell 1 . The simulation parameters used to obtain the numerical results are given in Table I.

Performance Metric: The performance metric considered for the numerical results is the normalized channel estimation error $\mathcal{E}$, which is defined in $\mathrm{dB}$ scale as

$$
\mathcal{E}[\mathrm{dB}]=10 \log _{10}\left(\frac{\left\|\hat{\mathbf{h}}_{n 11}-\mathbf{h}_{n 11}\right\|_{\mathrm{F}}^{2}}{\left\|\mathbf{h}_{n 11}\right\|_{\mathrm{F}}^{2}}\right) .
$$

SIMULATION PARAMETERS

\begin{tabular}{|c|c||c|c|}
\hline Parameter & Value & Parameter & Value \\
\hline \hline$L$ & 7 & $r_{\mathrm{S}}$ & $100 \mathrm{~m}$ \\
\hline$\eta$ & 2.5 & $\gamma_{\mathrm{SNR}}$ & $20 \mathrm{~dB}$ \\
\hline$\lambda$ & $0.1 \mathrm{~m}$ & $P$ & 50 \\
\hline$\sigma_{n}$ & 0.001 & $\tau$ & 10 \\
\hline$R$ & $1000 \mathrm{~m}$ & $D$ & $\lambda / 2$ \\
\hline$K$ & 50 & & \\
\hline
\end{tabular}

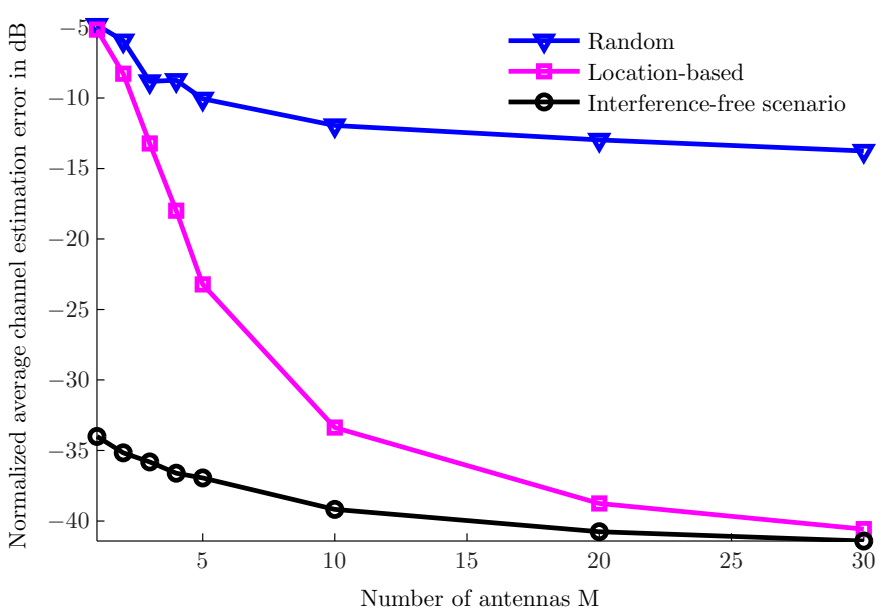

Fig. 2. Comparison of normalized average channel estimation error $\mathcal{E}$ versus number of antennas for random and location-based user selection methods. For each value of $M$, the results of $\mathcal{E}$ are averaged over 200 channel realizations.

Evaluation of Location-Based Assignment: In Fig. 2, the estimation error $\mathcal{E}$ versus the number of BS antennas is illustrated. It can be observed that $\mathcal{E}$ decreases with the increase in antenna number $M$ at the BS. This is achieved by exploiting the locations of users and BSs in obtaining the covariance matrices needed for the channel estimation. In the random assignment, $\mathcal{E}$ decreases initially and then becomes saturated in the large antenna regime. This is because it is not guaranteed that the AoA support of the interfering users is strictly nonoverlapping with the AoA support of the desired user. On the other hand, in the location-based user assignment, the overlap between the desired and interfering users is minimized. As a consequence, $\mathcal{E}$ decreases rapidly and approaches the interference-free scenario channel estimation performance in the large antenna regime.

Impact of Location Uncertainty: In practice, users may not have access to their true location as localization is subject to various sources of uncertainty. The accuracy of the location depends on the localization technology used and also on the environment (outdoor or indoor). We quantified the impact of the location uncertainty on $\mathcal{E}$ for location-based user assignment in Fig. 3. The user selection is done based on the estimated location information and while evaluating $\mathcal{E}$ the true channels are generated using actual location information. It can be seen that the location-based user selection is robust to small levels of location uncertainty, up to 10 meter, which 


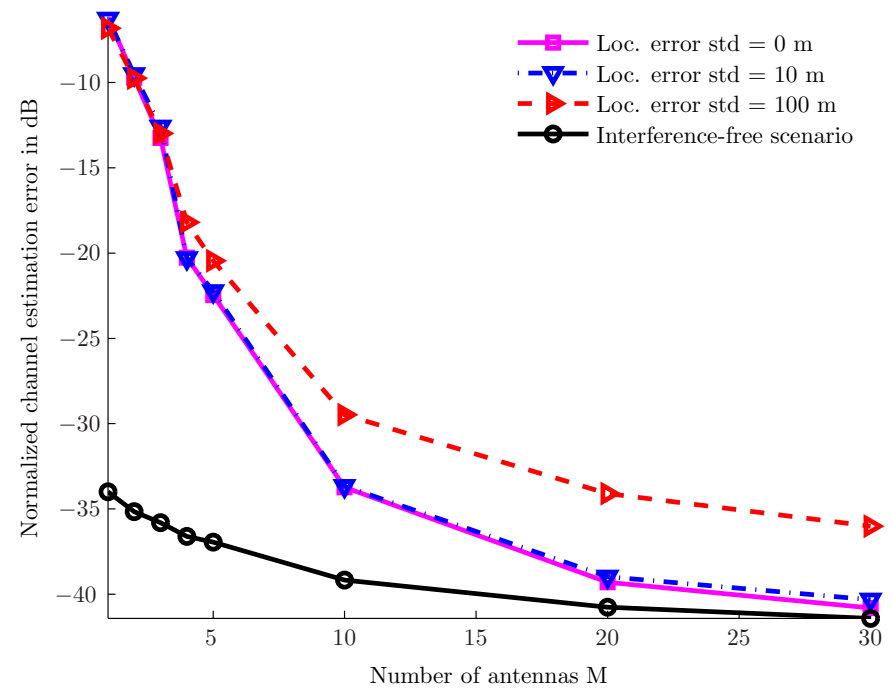

Fig. 3. Normalized average channel estimation error $\mathcal{E}$ versus number of antennas at BS for location-based user assignment with different levels of location error standard deviation. For each value of $M$, the results of $\mathcal{E}$ are averaged over 200 channel realizations and over 100 realizations of the location error.

is similar to the performance of commercial GPS receivers. On the other hand, when the location uncertainty is high (100 $\mathrm{m})$, the performance is degraded and requires more antennas to approach the interference-free scenario. The reason for this is that the user selection, which is done based on estimated locations, is quite different from that using the true locations under high location uncertainty. This leads to overlapping AoA between the desired and interfering users with true locations.

\section{CONCLUSION}

We proposed the use of location information in mitigating pilot contamination present in massive MIMO systems. We demonstrated the computation of the user channel covariance matrices based on location information, thus eliminating the need for estimating channel covariance matrices directly. We established a spatially separable user set based on location information rather than on processing channel covariance matrices. With the proposed location-aided method, we showed that the normalized channel estimation error of the desired user decays rapidly with the increase in antenna number and approaches the interference-free scenario in the large antenna regime. Avenues for future research include joint assignment of users within the cell, assignment across cells, and assignment under more complex AoA distributions.

\section{ACKNOWLEDGMENT}

This research was supported in part, by the European Research Council, under Grant No. 258418 (COOPNET). Discussions with Dr. Themistoklis Charalambous are gratefully acknowledged.

\section{REFERENCES}

[1] F. Boccardi, R. W. Heath, A. Lozano, T. L. Marzetta, and P. Popovski, "Five disruptive technology directions for 5G," IEEE Communications Magazine, vol. 52, no. 2, pp. 74-80, Feb. 2014.
[2] Y.-G. Lim, C.-B. Chae, and G. Caire, "Performance analysis of massive MIMO for cell-boundary users," arXiv preprint 1309.7817, 2013.

[3] E. G. Larsson, O. Edfors, F. Tufvesson, and T. L. Marzetta, "Massive MIMO for next generation wireless systems," IEEE Communications Magazine, vol. 52, no. 2, pp. 186-195, Feb. 2014.

[4] F. Rusek, D. Persson, B. K. Lau, E. G. Larsson, T. L. Marzetta, O. Edfors, and F. Tufvesson, "Scaling up MIMO: Opportunities and challenges with very large arrays," IEEE Signal Processing Magazine, vol. 30, no. 1, pp. 40-60, Jan. 2013.

[5] E. Bjornson, E. G. Larsson, and M. Debbah, "Optimizing multi-cell massive MIMO for spectral efficiency: How many users should be scheduled?" in Proc. IEEE Global Conference on Signal and Information Processing, Dec. 2014, pp. 612-616.

[6] T. L. Marzetta, "Noncooperative cellular wireless with unlimited numbers of base station antennas," IEEE Transactions on Wireless Communications, vol. 9, no. 11, pp. 3590-3600, Nov. 2010.

[7] J. Hoydis, S. Ten Brink, and M. Debbah, "Massive MIMO in the UL/DL of cellular networks: How many antennas do we need?" IEEE Journal on Selected Areas in Communications, vol. 31, no. 2, pp. 160-171, 2013.

[8] H. Q. Ngo and E. G. Larsson, "EVD-based channel estimation in multicell multiuser MIMO systems with very large antenna arrays," in Proc. IEEE International Conference on Acoustics, Speech and Signal Processing, Mar. 2012, pp. 3249-3252.

[9] V. Saxena, G. Fodor, and E. Karipidis, "Mitigating pilot contamination by pilot reuse and power control schemes for massive MIMO systems," in Proc. IEEE Vehicular Technology Conference Spring, May 2015.

[10] K. Appaiah, A. Ashikhmin, and T. L. Marzetta, "Pilot contamination reduction in multi-user TDD systems," in Proc. IEEE International Conference on Communications, May 2010.

[11] W. A. W. M. Mahyiddin, P. A. Martin, and P. J. Smith, "Pilot contamination reduction using time-shifted pilots in finite massive MIMO systems," in Proc. IEEE Vehicular Technology Conference (Fall), Sep. 2014.

[12] T. X. Vu, T. A. Vu, and T. Q. S. Quek, "Successive pilot contamination elimination in multiantenna multicell networks," IEEE Wireless Communications Letters, vol. 3, no. 6, pp. 617-620, Dec. 2014.

[13] J. Zhang, B. Zhang, S. Chen, X. Mu, M. El-Hajjar, and L. Hanzo, "Pilot contamination elimination for large-scale multiple-antenna aided OFDM systems," IEEE Journal of Selected Topics in Signal Processing, vol. 8 , no. 5, pp. 759-772, Oct. 2014.

[14] H. Yin, D. Gesbert, M. Filippou, and Y. Liu, "A coordinated approach to channel estimation in large-scale multiple-antenna systems," IEEE Journal on Selected Areas in Communications, vol. 31, no. 2, pp. 264273, Feb. 2013.

[15] R. Di Taranto, S. Muppirisetty, R. Raulefs, D. Slock, T. Svensson, and H. Wymeersch, "Location-aware communications for 5G networks: How location information can improve scalability, latency, and robustness of 5G," IEEE Signal Processing Magazine, vol. 31, no. 6, pp. 102-112, Nov. 2014.

[16] M. Bashar and D. Slock, "Cognitive multi-user MIMO downlink with mixed feedback/location based Gaussian CSIT," in Proc. 15th International Workshop on Signal Processing Advances in Wireless Communications, 2014, pp. 155-159.

[17] H. C. Papadopoulos, G. Caire, and S. A. Ramprashad, "Achieving large spectral efficiencies from MU-MIMO with tens of antennas," in Proc. 47th Asilomar Conference on Signals, Systems and Computers, 2010, pp. 636-643.

[18] A. Adhikary, J. Nam, J.-Y. Ahn, and G. Caire, "Joint spatial division and multiplexing: The large-scale array regime," IEEE Transactions on Information Theory, vol. 59, no. 10, pp. 6441-6463, Oct. 2013. 